\section{Der gegenwärtige Stand der Anschauungen über Entstehung und Einteilung der Nierenentzündungen.}

\author{
Von Prof. H. Straub in Berlin.
}

Wenn ich einer Aufforderung des Herrn Herausgebers dieser Wochenschrift folge und hier einen kurzen Ueberblick über den jetzigen Stand der Entstehung und Einteilung der Nierenentzündungen $\mathrm{zu}$ geben versuche, so will ich vorausschicken, daß ich hierbei nur solche Gesichtspunkte berücksichtigen werde, welche ein Interesse vom Standpunkt des Praktikers verdienen. Deshalb möchte ich hier nur solche Punkte in den. Vordergrund drängen, welclie für Di a gnose, Prognose und Therapie von Wichtigkeit sind, und Fragen ausschließlich theoretischen Charakters nur nebenbei erörtern. AuBerdem möchte ich noch besonders betonen, daB es nicht in der Absicht der vorliegenden Ausführungen liegt, eine irgendwie erschöpfende Erörterung des vorliegenden Gebietes zu geben. Entsprechend einer solchen Tendenz des vorliegenden Aufsatzes ist für den genannten Zweck auch noch die Ueberschrift "Nierenentzündungen" beibehalten worden, indem der Praktiker diesen Namen zur Zeit noch für cine ganze Reihe von Vorgängen und Zuständen anwendet, welche vom anatomischen Standpunkt aus nicht in das Kapitel der "Entzündung" gehören. Die Nierenentzündungen im engeren Sinne werden aber tür die vorliegenden, praktische Ziele ins Auge fassenden Betraclitungen den Stamm abgeben, um welchen andere Prozesse gewissermaften als Ranken gruppiert sind. Würde der Name "Nephropathie" nicht auch noch andere Nierenkrankheiten, welche nicht in den vorliegenden Interessenkreis gehören, umfassen, so wäre der Name "Nephropathie" vielleicht als eine zusammenfassende Bezeichnung für die hier interessierenden Vorgänge geeigneter. So wie die Dinge aber zur Zeit liegen, läßt sich der Name "Nephropathie" nur verwenden, wenn durch ein ihm beigegebenes Adjektivum die spezielle Art der Erkrankung angezeigt wird, welche Gegenstand der Betrachtung sein soll (Aschoff u. a.). Gewiß wäre es erheblich leichter, das hier zur Erörterung stehende Gebiet zu besprechen, wenn die Pathologische Anatomie und die klinischen AeuBerungen der verschiedenen hier $\mathrm{zu}$ erörternden Nierenerkrankungen enen durchgreifenden Parallelismus zu ihrer Entstelıung besitzen würden. Dies trifft aber leider nur in etwas beschränkter Weise zu. Denn wir kennen nicht nur eine ganze Reihe von Ursachen, welche verschiedenartige anatomische Veränderungen an den Nieren zu erzeugen vermögen, sondern wir wissen auch, daß ein und dieselbe Veränderung an den Nieren durch eine ganze Reihe verschiedener Ursachen erzeugt werden kann. Dazu kommt noch, daß die für die klinische Erscheinungsweise der Krankheit maßgebenden Veränderungen an den Nieren nicht bloß von der Art, sondern auclı von dem Grad der die Krankheit erzeugenden Ursache abliängen.

Das Gesagte ergibt sich schon, wenn wir die harmlosen, vorübergehenden Albuminurien von den echt nephritischen oder nephrotischen Prozessen abzugrenzen versuchell. So leiclit eine solche Abgrenzung in vielen Fällen gelingt, so findet sie doch in zahlreichen anderen Fällen deslıalb groß̉e Schwierigkeiten, weil sich die Uebergänge zwischen vorïbergehenden Störungen und länger dauernden Veränderungen an den für die Albuminurieerzeugung in Frage kommenden Stellen der Nieren als sehr fließend erweisen. Ganz allgemein kann man unter den ätiologischen Momenten neben lokal wirkenden Ursachen Ernährungsstörungen auf zirkulatorischem Wege und auf toxischem bzw. toxisch-infektiösem Wege unterscheiden. Die Zahl der zur letzteren Gruppe gehörigen Nexen ist überaus groß, Ist doch bei ihr neben der großen Zahl von exogen wirkenden Schädlichkeiten auch eine nicht geringe Anzahl von endogen entstandenen Noxen zu berücksichtigen. Die anatomischen und klinischen Bilder, die hierdurch erzeugt werden können, hängen dabei nicht bloß von der Intensität und Akuität der Noxe, sondern auch von dem Angriffspunkt der Noxe ab. Prinzipiell kann man in bezug auf den letzteren tubulotrope und vaskulotrope Noxen unterscheiden, wobei unter vaskulär das Vas afferens einschließlich des zugehörigen Glomertulus verstanden sein soll. Ihnen gesellt sich allerdings erheblich seltener - noch eine dritte Gruppe voll Veränderungen $z u$, die im eigentlichen "Interstitium", d. h. in dem Zwischengewebe, welches die Gefäße und das Parenchym der Nieren zusammenhält, Platz greifen. Die Ernährungsstörung an den Epithelien kann entweder in trüber Schwellung bzw. in fettiger oder lipoider oder auch in nekrotisierender Degeneration bestehen. Am
Vaskularapparat können wir außer entzündlichen Prozessen auch noch hyaline und amyloide Degeneratiouen unterscheiden. Für die klinischen Aeußertungen des Proresses ist ferner noch die Frage von Wichtigkeit, ob die Niere in ihrer $T$ ot alität oder nur an einzelnen Stellen , insulär" in Form einer Partialaffektion erkrankt ist. Je nach dem Weg, den die Noxe genommen hat, darf man hänuatogene und vom Lumell der Ausführungsgänge aus wirkende „aszendierende" Nierenschädigungen unterscheiden. Es wäre aber unzureichend, nur die hier genannten Gesichtspunkte ins Auge zu fassent. Denn die verschiedenen Nieren sind derselben krankmachenden Ursache gegelliiber in verschiedenem Grade anfällig. Hierfür sind verschiedene ,konstitutionelle" und ", konditionelle" Faktoren (s. später) maßgebend. Von den letzteren sei hier nur die Erkältung genannt, die auch jetzt noch für die Entstehung akuter Prozesse als wirksamer Faktor vielfach angeschuldigt wird. Man darf dieser tatsächlich auch häufig die Eigenschaft eines "Schrittnachers" nachsagen. Meist liegen die Dinge aber so, daß erst das Zusammenwirken einer Mehrheit von $\mathrm{Faktoren}$ das Zustandekommen der anatomischen Verände. rungen und der klinischen Bilder bewirkt.

Wenn man unter den hier genannten Gesichtspunkten die Vielgestaltigkeit der uns entgegentretenden anatomischetı und klinisclien Bilder betrachtet, so besitzen wir zwar ke in Schema und keine bündige Formel, welche uns in allen Fällen eine Orientierung crmöglicht, wohl aber gewisse Richtlinien, die sich daraus ergeben, da $B$ in den ausgeprägten Fällen verschiedene, bis zu einem gewissen Grade übereinstimmende Symptomenkomplexe für bestimmte anatomische Veränderungen vorhanden sind und daß nach der $\mathrm{Er}$ fahrung eine Reihe von Noxen mit einer gewissen Vorliebe ganz bestimmte anatomische Veränderungen und demgemäß auch bestimmte klinische Bilder 7.4 erzeugen pflegen. Aus den eingangs angegebenen Gründen sollen jedoch aus der Summe der hier zur Erörterung stehenden Befunde nur die markantesten, für die Beurteilung der Nierenentzündung en verwertbaren Feststellungen erörtert werden. Ergibt sich doch hierdurch schon, daß durch den modernen Ausbau der Pathologischen Anatomie und der Diagnostik der Nierenerkrankungen die Uebersicht nach vielen Richtungen erleichtert worden ist.

Dies zeigt sich schon bei einer Betrachtung des viel erörterten und viel umstrittenen Gebietes der, $z y k$ lischen $b z w$. inter. mittierenden" Albuminurie. lan der weitaus überwiegenden Mehrzahl der vorliegenden Fälle handelt es sich unseres Erachtens um cine Ernährungsstörung am Epithelialapparat, die in der Regel durch eine nur kurzdauernde Giftwirkung oder durch eine durch lokale Momente (Ischämie oder venöse Stauung) oder durch allgemeine Momente (Einwirkung der Ermüdung auf die Blutversorgung der Nieren) bedingte, jedenfalls vorübergehiende Ennährungsstörung erzeugt ist. Rechnet man in die vorliegende Gruppe auber der "orthotischen oder lordotischen" Albuminurie auch noch die ver"schiedenen "Insultalbuminurien" (so z. B. die Marschalbuminurie, die Albuminuria intra partum, die Narkosenalbuminurie usw.), so läßt sich eine scliarfe zeitliche Umgrenzung des Begriffes der Albuminuria "fugax" nicht geben, und wir finden hier bei der Abgrenzung dieser Gruppe fließende Úebergänge zu kurzdauernden Infektionsalbuminurien und $\mathrm{zu}$ gewissen seltenen Formen von neurogener Albuminurie. Bei den hier zur Erörterung stehenden Formen zeigt sich in besonderem Grade, daß eine Erklärung der Erscheinung ohne ein "konditionelles" Denken nicht möglich ist, indem cin und dieselbe Noxe in dem einen Fall mit tund in dem anderen Fall ohne Albuminurie verläuft und indem wir auch den Grad und die Zeitdauer der Albuminurie bei den verschiedenen Personen trotz der Gleichartigkeit der einwirkenden Ursache durchans wechseln sehen. Für das Zustandekommen der Erscheinung ist also auch hier schon eine Mehrheit von Momenten, insbesondere auch ein bestimmter präexistierender Zustand der Nieren ins Auge zu fassen, wie er teils in angeborenen "konstitutionellen" Momenten, teils in erworbenen "kouditionellen" Momenten, so $u$. a. auch in vorausgegangencr Abnutzung oder in Veränderungen durch voransgegangene Erkrankungen, insbesondere abklingende akute oder geringgradige chronische Nephritiden, gegeben ist. Neben der eigentlichen, die Krankheit bestimmenden Ursache ist also lier oft auch eine "Disposition"s, eine "Bereitschaft", eine „Empfindlichkeit" der Nieren z.u berücksichtigen. Schon hier gilt die Gottstein-, Martius.

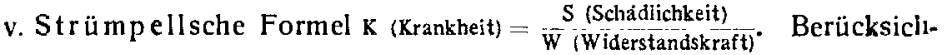
tigt man außerdem noch die Bedeutung des graduellen Faktors, so wird man sich nicht wundern, daß eine Albuminuria "fugax" auf Grund derselben Ursache das eine Mal nur wenige Stunden, das andere Mal ein oder mehrere Tage anhalten kann. $\mathrm{Da}$ die $\mathrm{Er}$ fahrung gelelirt hat, daB bei bestehender Albuminuriebereitschaft unter Umständen auch stärkere Grade von transitorischer Albuminurie erzeugt werden können und $\mathrm{da} \beta$ bei stärkeren Graden von Nierenkongestion und entsprechender Durchlässigkeit der Gefäße auch beim Fehlen von echt entzündlichen Prozessen - so z. B. in manchen Formen von Sport- bzw. Ermüdungsalbuminurie - aucl Erythrozyten in größerer Anzahl itı den Urin übertreten können, so haben sich ganz eindeutige, stets zutreffende Formeln für die Abgrenzung eines bestimmten Urinbildes im Sinne der Albuminuria fugax bzw. der zyklischen ader orthotischen Albuminurie, d. l. im Sinne einer Albuminuria ,innocua", nicht aufstellen lassen. Wissen wir doch ferner, da $\beta$ auch bei passageren harmlosen Ernährungsstörungen der Nieren Zylinder in verschiedenen Formen vorkommen können. 
Auch hier ist zur Stellung der Differentialdiagnose eine Sum me von Feststellungen innd Erwägungen mötig, die sich außerdem noch nicht bloB auf die Betrachtung des Urins grürnden dürfen.

Will man überhaupt die Menge des ausgeschiedenen Eiweißes als Ausgangspunkt für die Beurteilung bestimmter pathologischanatomischer Vorgänge benutzen, so darf dies nur bei exzessiven Graden von Albuminurie und unter gle ich ze itig er vollster Berücksichtigung der klinischen Eigentümlichkeiten des Falles geschehen. Nur sehr hohe Grade von Albuminurie sind geeignet, an sich schon auf eine Erkrankung des $E_{p}$ ithellia l'a p parates hinzuweisen, und dies in um so höherem Grade, je mehr im Urin eine Beimengung von Erythrozyten fehlt. Beobachten wir doch bei $E_{r}$ krankungen, bei welchen uns die Pathologische Anatomie ein ausschlieBliches oder-neben anderen Veränderungen gleichzeitig thervortretendes Befallensein des Epithelialapparates aufdeckt, so z. B. bei der Quecksilbervergiftung, bei Amyloid, bei bestimmten Formen von Nierensyphilis, oft exzessive Grade von Albuminurie, ohne dab im Zentrifugat des Urins nennenswerte Mengen von Erythrozyten zu finden sind. Die Diagnose einer Tubularerkrankung gewinnt dabe noch an Sicherheit, wenn im Urin Nierenelemente und solche Zylinder zu finden silld, deren Entstehung wir in eine Beziehung zur Abstoßung oder zum Untergang von Nierenelementen zu bringen gewohnt sind. Für die Lokalisation des Krankheitsprozesses an den kleinen Gefäßen und an den Glomeruli spriclit dagegen das Vorhandensein größerer Mengen von Erythrozyten im Urin, obwohl auch hier die Erfahrung lehrt, daB auch im interstitiellen Gewebe liegende Blutextravasate gelegentlich in die Ausführungsgänge der Nieren durchbrechen können. Für $m$ a $n$ ch e Fälle von Vaskularerkrankungen der Nieren besitzt ferner auch die Hypertonie den Charakter eines wertvollen Symptoms. Aber auch diesem Symptom kommt llur eilt bedingter Wert zu, indem dieser mehr von den Wirkungen als von der Eigenart des anatomischen Prozesses abhängt. Sehen wir doch nicht nur zahlreiche Vaskularerkrankungen der Nier dauernd oder zeitweilig ohne Blutdrucksteigerung einhergehen, sondern wir finden das Symptom doch gelegentlich anch bei reitten Tubularerkrankungen, wenn es bei solchen zu länger dauernder Oligurie oder Anurie gekommen ist. Außerdem fillden wir das Phänomen auch noch bei der sogenannten "essentiellen" Hypertonie, d. h. einem Zustande, bei welchem es sich um eine Erkrankung des Präkapillarapparates des gesamten Körpergefäßsystems, um eine sogenannte Arteriolosklerose handelt, also unter ganz anderen Bedingungen als bei der nephrogenen. Hypertonie, welche auf eine mangelhafte Ausscheidung hypertonisierender Substanzen bzw. deren Wirkung auf die Vasomotoren zurückgeführt werden darf. Will man das Symptom der Hypertonie im Dienste der Nierendiagnostik überhaupt benützen, so müssen gleichzeitig auch Zeichen für dessen nephrogene Entstehung vorliegen. Da wir aber aus der Erfahrung wissen, daß es beim Fehlen von Anzeichen eilter schweren, mit länger datiernder Oligurie einhergehenden Erkrankung des Parenchyms fast nur bei Vaskularerkrankungen vorkommt und daß letztere nur dann zu Retentionen führell, wenn sie einell stärkerell Grad und eine diffus über die Nieren verbreitete Ausdehnung besitzen, so ist das Symptom der Hypertonie - - auch in dieser Beschränkung - recht wertvol geworden. Unter ähnlichen Gesichtspunkten wie die Hypertonie is auch die Stellung der Herzliypertrophie vom anatomischen und klinischen Standpunkt zu beurteilen. Aucl das Symptom der $\mathrm{Hy}-$ dropsiebildung besitzt in älnlicher Weise einen nur bedingten Wert. Selbst wenn man, was absolut notwendig ist, den kardiogenen Hydrops scharf von dem nephrogenen Hydrops unterscheidet, ist nur der positive Befund des letzteren für die differentialdiagnostischen Zwecke verwelldbar, und zwar in der Form, daß ein nephrogener $\mathrm{Hy}$ drops auf eine Erkrankung des Epithelialapparates hinweist. Auch die Entstehung des Hydrops nephroticus hängt von einer Meltrheit vor Faktorell ab. Doch soll hierauf nicht näher eingegantgen werden, da hier nur seine klinisch- bzw. topisch-diagnostische Verwertbarkeit zur Erörterung steht. Von dell Ergebnissen der sogenannten $\mathrm{F}$ unktionsprüf ung weist eine Erhöhung der Werte für del Reststickstoff auf eine Störulg am Vaskularapparat hin, falls nicht hochgradige Oligurie oder Allurie bei scltweren Parenchymstörungen 211 einer beträchtlichen Gesamitretention Anlaß gegebell haben. Je "lach der Höhe des Wertes für den Reststickstoff können wir ,azotämische" und ,,anazotämische" Zustände unterscheiden. Wir sprechen hier nur von "Zuständen", weil auch der Reststickstoffgehalt - ähn. lich wie auch das Symptom "Hydrops“ _- im gleichen Fall zeitlicl wechseln kann. Auch das Verhalten der Uriumenge, des spezifischen Gewichtes und des Kochsalzgehaltes des Urins bei Anstellung des "Akkomodationsversuclyes" vermag wertvolle Fingerzeige einerseits für topisch-diagnostische Zwecke, anderseits für die Feststellung einer Suffizienz oder Insuffizienz der Nicren zu geben, doch lıängt die semiotische Bedeutung der Ergebnisse der Urinuntersuchung beim "Akkonıodationsversuch" voll der Berücksichtigung auch' noch anderer Befunde ab. Für die Einteilung und Beurteilung der einzelnen Formen von Niereneutzülldung sind außerdem aber auch noch ganz allgemein die Art des Verlaufs und die Bedingungell, unter welchen die Krankheit entstanden ist, mitzuverwertell.

Für die Unterscheidung der eilızelnen Forme 11 kommt man im allge in einen mit einer $Z$ weiteilung aus, indem man akute und chronische Prozesse, lıerdförmige (insuläre) und diffuse, tubuläre und vaskuläre Prozesse unterscheíden kanı. Als a kut bezeichnet man dabei diejenigen Prozesse, dic akut be gonnen haben, als chronisch solche. welche sich entweder aus akuten entwickelt haben und schon sehr lange datuern oder welche von vornherein schleichend eingesetzt haben.

Die herdförmigen Prozesse kann man grundsätzlich in zwei Gruppen einteilen, in solche Prozesse, welche am Vaskularapparat sitzen, und in solche, welche im interstitiellen Gewebe gelegen sind. $\mathrm{Ob}$ es auch primäre insuläre Veränderungen am Epithelialapparat gibt, laßt sich schwer entscheiden. Sicher muB man aber das Vorkommen sekundärer insulärer Veranderungen am Epithelialapparat annehmen, da die Versorgung der einzelnen Tubuli vorwiegend vom Vas afferens des zugehörigen Glomerulus stattfindet (Löhlein) und da man außerdem auch mit der Möglichkeit rechnen muB, daß bei interstitiellen Prozessen das benachbarte Parenchym mit ergriffen werden kann. Beide Fälle stellen aber nicht selbständige Vorgänge, sondern nur Folgeerscheinungen eines der hier genannten Grundprozesse dar. Von dell akuten ins uIären Prozessen sind die eitrige metastatische und die blande embolische Glomerulonephritis (insbesondere nach Endocarditis lenta) wohl charakterisiert, daneben wird von Volhard und $F$ ahr aber auch noch eine herdförmige nicht bakterielle Glomerulonephritis angenommen. Die chronischen insulären Prozesse sind durch die embolisclie Schrumpfniere nach Niereninfarkten und die senile arteriosklerotische Schrumpfniere ( $Z$ i egler) repräsentiert. Daneben mögen auch noch chronische insuläre Entzündungsprozesse berücksichtigt werden, wie man sie bei der "Nephrite parcellaire“ bzw. der "Néphralgie hématurique" antrifft.

Die diffusen Nierenentzündungen lassen sich in $h$ istologischer Hinsicht in degenerative und entzündliche einteilen. Stellt man sich auf den Standpunkt, daß die arteriosklerotischen Prozesse in der Niere als degenerative Vorgänge an den Gefäßen zu rubrizieren sind, so kann man diese Prozesse als Untergruppe der vaskulären Nierenveränderungen registrieren und auf diesem Wege an Stelle eines Trialismus einem Dualismus huldigen Die arteriosklerotischen Prozesse sind aber klinisch und anatomisch von dem, was man Entzündung nennt, so wesensverschieden, daß es zweckmäBig erscheint, diese Gruppe, bei welcher ja die Nieren auch nur einen Teil der anatomischen, an verschiedenen Stellen des Gefäßsystems entwickelten Veränderungen darstellen, von den übrigen hier zur Erörterung stehenden Prozessen völlig abzutrennen. Sieht man also bei einer Einteilung von den arteriosklerotischen Prozessen und auch voll der Stauungsniere ganz ab, so kann man bei den vaskulären bzw. glomerulären Prozessen grundsätzlich eine akute und chronische Form unterscheiden und mit Rücksicht auf den entzündliclıen Charakter derselben von akuten oder chronischen "N e phritiden" bzw. "Glomerulonephri-

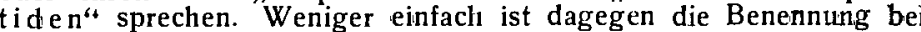
den Tubularerkrankungen. Hier trifft eine Zusammenfassung schon auf gewisse Schwierigkeiten, je nachdem man mit Ribbert t. a. auf dem Standpunkt steht, daß alle in Frage kommenden Epithelialerkrankungen als degenerative $\mathrm{zu}$ betrachten sind, oder mit Virchow und Aschoff annimmt, daß es auch eine parenchymatöse Entzündung gibt. Außerdem ist der für die vorliegende Gruppe gewähite Name "N ephros " von den einzelnen Autoren in nicht ganz gleicher Weise gebraucht worden. So hatte ihn Vierenerkrankungen vorgeschlagen und Volhard ihn zunächst für die große weiße Niere, so weit diese nicht sekundär aus einer primären Glomerulonephritis hervorgegangen war, gebraucht. Vol ha rd hat ihn aber auch noch im weiteren Sinne angewandt, indem er sagt „Für den Histologen fallen unter dell Begriff der degenerativen Nephropathien nicht nur diejenigen der bisher als parenchymatöse Nephritiden bezeichnete Formen, die ohne Blutdrucksteigerung und ohne Glomerulitis verlaufen, sondern alle degenerativen Nierenveränderungen überhaupt, die Nekrose, die trübe Schwellung, die hyalin-tropfige Entmischung und die Verfettung. Klinisch bietell aber nur die beiden letztgenannten degenerativen Veränderungen das bekannte Bild der sog. chronisch-parenchymatösen Nephritis, der Nephrose im engeren klinischen Sinne." Für eine weite Fassung ganz allgemein im Sinne einer degenerativen Tubularerkrankung hat sich auBer ınir auch $F$. Munk entschieden. Zählt man aber bei einer Einteilung auch die diffusen arteriosklerotischen bzw. arteriolosklerotischen Erkrankungen mit, so kann man diese Prozesse, wie schon bemerkt ist, entweder als eine degenerative Untergruppe der vaskulären Erkrankungen rubrizieren, oder man muB sich zur Wahrung ihrer selbständigen Stellung zu einer Dreiteilung des Gebietes entschließen, wie es Volhard tat, indem er grundsätzlich degenterative Erkrankungen, entzündliche Erkrankungen und arteriosklerotische Prozesse an dell Nieren unterschied In der Gruppe der "Schrumpfnieren“ ist, falls wir von den arteriosklerotischen Nierenerkrankungell absehen, die en tzündlich Form (,sekundäre" Schrumpfniere) von den überaus seltenen, ohne Hypertonie verlaufenden "nephrotischen" Schrumpfnieren zu unterscheiden. Diesen stehen dann die angiogenen, originären, auf dem Boden einer diffusen Arteriolosklerose entstandenen, Schrumpfnieren gegenüber, bei welchen wir, je nach ihrem Verlauf und dem Vorhandensein oder Fehlen von Retentionen, eine langsam verlaufende gutartige und eine rasch progrediente bösartige Form unterscheiden können. Im ganzen wird aber in der Klinik der anatomische Begriff Schrumpfniere heute nicht mehr so häufig gebraucht als früher, und zwar auch schon deshalb, weil wir auch

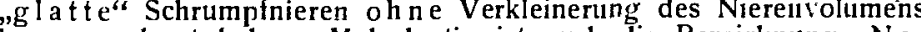
kennen gelernt haben. Mehrdeutig ist auch die Bezeiclınıng , $\mathrm{Ne}$ - 
phrosklerose“, indem manche Autoren diesen Begriff nur für arteriolosklerotische Nierenerkrankungen reservieren, während ihn andere ganz allgemein mit Schrumpfniere indentifizieren.

Eine klinische und anatomische Einteilung der hier zur $\mathrm{Er}$ örterung stehenden Nephropathien wäre viel leichter, wenn $M$ is ch formen nicht so überaus zahlreich und vielgestaltig wären. Vom klinischen Standpunkte werden die Bilder außerdem noch dadurch sehr kompliziert, daß die Aeußerungen der Krankheit verschieden sind, je nachdem es sich um kompensierte oder nicht kompensierte Fälle handelt; wobei man hinsichtlich der Kompensationsstörung außer einer $k$ ardialen Dekompensation auch noch eine renale Dekompensation zu unterscheiden hat. Unter renaler Kompensation sind dabei alle die Vorgänge einzubegreifen, die bei Kostörter Nierentätigkeit das Auftreten von Retentionen bzw. bei Vorhandensein der letzteren eine schädigende Wirkung derselben auf den Gesamtorganismus zu verhüten vermögen. Infolgedessen können und müssen wir in den verschiedenen Gruppen noch e udynamische und adynamische Fälle unterscheiden. Soweit die diffusen rein arteriolosklerotischen Formen in Frage kommen, hat Volhard die ersteren als benigne oder blande Nephrosklerosen den malign e $n$ gegenübergestellt. Hinsichtlich der g e $n$ etischen Unterscheidung sind bereits die hämatogenen und aszendierenden-urinogenen Formen einander gegenübergestellt. Formen von vorwiegend $n$ e phrot is chem Charakter trifft man besonders bei der Sublimatintoxikation, bei Cholera, Diphtherie, Scharlach sowie in manchen Fällen von Syphilis, Tuberkulose und nach schweren Wundinfektionen, Formen von vorwiegend vaskulärem Charakter findet man besonders nach Scharlach, Angina, Endokarditis, Osteomyelitis, Influenza, Masern, Erysipel, Gelenkrheumatismus, Typhus, Paratyphus und Koliinfektionen, Pocken, Varizellen, Pertussis, sowie in manchen Fällen von Syphilis und Tuberkulose. Durch die Vielheit der hier erörterten. Faktoren und auBerdem noch durch das Fehlen oder Vorhandensein von Komplikationen wird den einzelnen Fällen von Nephri. tis ein besonderes Gepräge verliehen, sodaß man sich nicht wundern wird, wenn es nur in einer begrenzten Anzahl von Fällen gelingt, das Wesentliche des Falles in ein ohne weiteres das Charakteristische des Falles wiedergebendes Schlagivort zu fassen. So gro $B$ auch die Fortschritte sind, welche Klinik und Pathologische Anatomie in den letzten 20 Jahren auf dem vorliegenden Gebiete gemacht haben, so ist doch auch hier - ähnlich wie auf vielen anderen Gebieten der Inneren Medizin - e in e g en a u e Beurteilung des Einzelfalles schließlich nur auf Grund eime Summe von Betrachtungen und Erwägungen möglich, die in zahlreichen Fällen von verschiedenen Gesichtspunkten gleichzeitig ausgehen müssen. Diese durch die Verhältnisse er2wungene Notwendigkeit der Betrachtung ist aber für die Zwecke der Therapie oft direkt ein Vorteil, indem für eine individualisierende Betrachtung des Einzelfalles eine mehrere Eigenschaften des Falles ausdrückende Benennung oft wertvoller ist als die schablonenunäBige Unterbringung in ein nur wenige Rubriken enthaltendes Schema, so hoch die Bedeutung eines solchen auch als eines Wegweisers für die Ordnung des ganzen Gebietes und als eines Anhaltspunktes für die Untersuchung des einzelnen Falles angesehen werden muß. So freudig auch eine klare Disposition des Gebietes begrüßt werden muß, so ist doch dic Fülle und die Mannigfaltigkeit dessen, was die Natur bietet, so groß daB eine solche Disposition für die Beurteilung der einzelnen Fälle, wie bereits gesagt ist, nur die Form von "Richtlinien" gewinnen kann. 